\title{
PROPAGATION OF POSITIONAL MEASUREMENT ERRORS TO FIELD OPERATIONS
}

\author{
S. de Bruin ${ }^{\text {a, } 1}$ G.B.M. Heuvelink ${ }^{\text {a }}$ J.D. Brown ${ }^{\text {b }}$ \\ ${ }^{a}$ Wageningen UR, Wageningen, The Netherlands - (sytze.debruin, gerard.heuvelink)@wur.nl \\ ${ }^{\mathrm{b}}$ National Weather Service, N.O.A.A., Silver Spring, MD, USA - james.d.brown@noaa.gov
}

KEY WORDS: Positional accuracy, GPS, vector data, stochastic simulation, precision agriculture

\begin{abstract}
:
Precision agriculture requires detailed information about numerous factors that determine optimal agricultural management. Recently, the importance of accurate assessment of field boundaries for precision agriculture has also been recognised. It has been claimed that the upcoming targeted approach to managing field operations requires that field boundaries are measured with $\mathrm{cm}$ level accuracy, thus avoiding losses such as wasted inputs, unharvested crops and inefficient use of the area. The aim of this work is to develop a method for experimental verification of such claims. The method comprises three main steps. The first step concerns the choice and parameterisation of a geostatistical model defining positional error. Secondly, the model is used to generate a set of possible realisations of field geometry. Next, these realisations are the input to a model computing the effects of positional uncertainty on field operations. The output is compared with that obtained using reference geometry. We illustrate the method using, amongst others, data from the 2005 GPS workshop of the EU Joint Research Centre. Our implementation of the model uses the Data Uncertainty Engine (DUE), which is free software that aids the user in defining probability distributions for uncertain spatial objects and draws random samples from these distributions. The application concerns an irregularly shaped field of approximately 15 ha. Results for three different measurement scenarios (Egnos augmented GPS, RTK-GPS, topographic map) are shown.
\end{abstract}

\section{INTRODUCTION}

The use of GIS and GPS in agriculture has increasingly moved from research to practical application. For example, in the Hoeksche Waard in the Netherlands, farmers are ready to invest in Real time kinematic (cm-accuracy) GPS technology (RTKGPS) and peripheral devices to support optimal allocation of field margins, vehicle path planning, variable rate application and other agricultural operations. A few years ago, the equipment required for these operations was very expensive and often required extensive customization of machinery (Keicher and Seufert, 2000). Nowadays, standard solutions are becoming available and costs are decreasing (Hekkert, 2006), thus improving the feasibility of GPS assisted farming (Nijland, 2006). In addition to these applications, GPS is used to validate the agricultural subsidies claimed by farmers who, based on topographic field boundaries, apply for money under the European Union Common Agricultural Policy (CAP). For this purpose, less accurate hand-held receivers are being used (Bogaert et al., 2005).

It is widely agreed that site-specific management, also known as precision agriculture, requires detailed information on soil and environmental attributes, such as texture, organic matter content, nutrient concentrations and incidence of diseases, weeds and pests (Atherton et al., 1999). Recently, however, the importance of accurate geometric positioning for the development of field operation maps has also been recognised (Earl et al., 2000; Gunderson et al., 2000; Choset, 2001; Fountas et al., 2006). It has been claimed that the upcoming targeted approach to managing field operations requires field boundaries to be measured with $\mathrm{cm}$ level accuracy, thus avoiding losses such as wasted inputs, unharvested crops and inefficient use of the area.
The aim of this work is to demonstrate a method for experimental verification of such claims. It employs positional error models and random sampling from these models (Monte Carlo) to assess error propagation from GPS measurements or digitized vertices along field boundaries through the planning procedure. We demonstrate the approach using error models based on three measurement scenarios, namely: (1) using handheld GPS with EGNOS (European geostationary navigation overlay service) correction; (2) using RTK-GPS measurements; and (3) based on a topographic vector product. The simulations were performed using reference geometry of an irregularly shaped field of approximately 15 hectares located in the Hoeksche Waard (see Figure 1)

Note that our analysis only considered positional uncertainty of mapped fields; semantic differences between topographic fields (which may have boundaries in the centre of ditches) and cultivated fields were not accounted for.

\section{METHODS}

\subsection{Error models}

The $\left(x_{i}, y_{i}\right)$ coordinates in the Dutch grid system of the $n=14$ corner points $(i=1 . . n)$ of the agricultural field shown in Figure 1 were measured by a professional surveyor using RTK-GPS equipment. The resulting coordinates and mapped field boundaries were used as the reference geometry in the present work. By construction, any observation error in these locations is of no consequence for our results (because the reference geometry constitutes our 'true' geometry in all subsequent calculations).

\footnotetext{
${ }^{1}$ Corresponding author.
} 
Under a measurement scenario, however, the coordinates of vertices (e.g. corner points) are subject to observational error which can be represented by the random variables $X$ and $Y$, with marginal cumulative probability distribution functions (mpdfs) $F_{X}$ and $F_{Y}$ (Eq. 1):

$$
F_{X}(x)=P(X \leq x) \quad \text { and } \quad F_{Y}(y)=P(Y \leq y)
$$

where $x$ and $y$ are real numbers and $P$ denotes probability.

The random variables $X$ and $Y$ typically have means (expected values) $\mu_{X}$ and $\mu_{Y}$ providing information on positional bias and standard deviations $\sigma_{X}$ and $\sigma_{Y}$, which are measures of spread in $x$ and $y$ direction, respectively. In the two-dimensional case, description of the positional uncertainty of a deformable object composed of $n$ vertices requires a $2 n$-dimensional joint probability density function (jpdf) which describes all mpdfs of the individual vertices together with all (cross) correlations (Eq. 2):

$$
\begin{aligned}
& F_{X_{1} Y_{1} \cdots X_{n} Y_{n}}\left(x_{1}, y_{1}, \ldots, x_{n}, y_{n}\right)= \\
& P\left(X_{1} \leq x_{1}, Y_{1} \leq y_{1}, \ldots, X_{n} \leq x_{n}, Y_{n} \leq y_{n}\right)
\end{aligned}
$$

Estimation of equation (2) typically relies on the assumption of second order stationarity, and on assumptions regarding the shape of the bivariate distribution and the function of statistical dependence (Heuvelink et al., 2007).

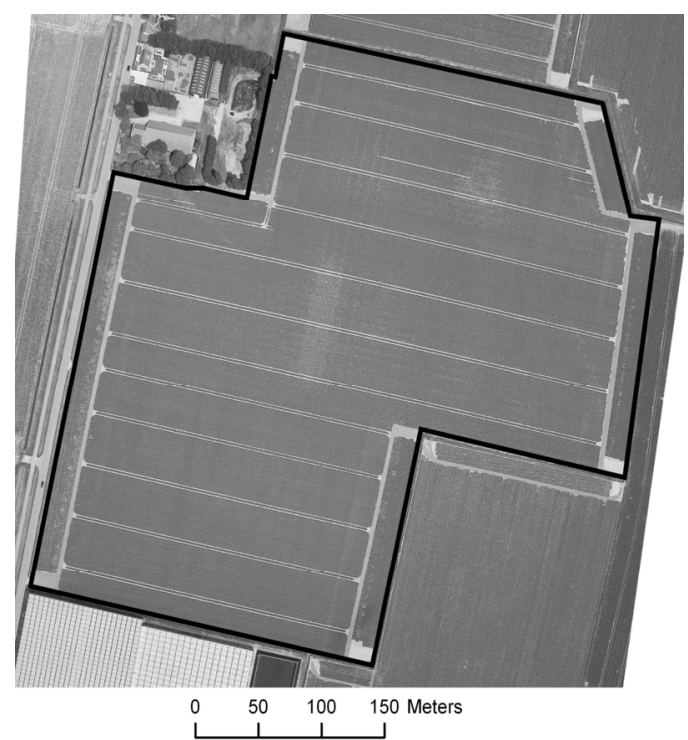

Figure 1. Potato field in the Hoeksche Waard; the black boundaries represent reference geometry; the greyish stripes are spray paths.

While geostatistical error models usually consider spatial correlation among observations, temporal variations in satellite clock errors, orbit errors, atmospheric delays and filtering by the GPS receiver itself may result in temporal correlation of observed positional errors (Olynik et al., 2002; Tiberius, 2003). Likewise, manual digitisation of polygons is a sequential procedure that is likely to result in temporal correlations among errors of vertices. Therefore, our error model considers temporal correlation of positional errors, which was described by semivariograms (see below). Clearly, these temporal correlations will lead to spatial correlations in the positional errors, but are better modelled as temporal correlations.

Similar to Bogaert et al. (2005), we assumed the errors to be normally distributed. However, unlike that work, we allowed for different variances for the GPS errors in $x$ and $y$ direction. In this context, the GPS satellite orbits cross the equator with an angle of $55^{\circ}$, which reduces the signal availability from the northern $(y)$ direction in the Netherlands $\left(52^{\circ} \mathrm{N}\right.$ latitude).

EGNOS provides GPS correction data and satellite integrity messages which improve the accuracy of GPS positioning (European Space Agency, 2004). In October 2005, during the GPS workshop of the EU Joint Research Centre, EGNOS was still in the test bed phase (European Space Agency, 2006).

A team operating Thales MobileMapper receivers provided us with time series of EGNOS augmented positional data, which were acquired to determine the area of three agricultural fields (Joint Research Centre, 2005). The positions were acquired at 1 s. interval, while the operator walked along pickets for which accurate RTK-GPS coordinates had been recorded. Some observations were removed by automatic filtering within the receiver. Each field was measured 10-14 times, but only the EGNOS augmented data were used in our analysis. Depending on the size of the field and the speed of the operator, a time series of GPS positions comprised 225 to $613 \mathrm{~s}$ of data. The errors in the $x$ and $y$ directions were defined as the differences between the EGNOS positions and the nearest point on the line segments connecting the pickets. The final dataset consisted of 10,839 error pairs.

Temporal dependence of the $x$ and $y$ errors were assessed by semivariogram analysis using Gstat (Pebesma and Wesseling, 1998). We used MobileMapper product information (Magellan, 2007) to set reasonable sills for the semivariograms. The data of all EGNOS augmented measurements were pooled, but temporal dependence between repeated measurements of the same field and between different fields were not analysed. We also assessed possible bias in the errors.

The thus parameterised error model was used to illustrate the positional uncertainty in field measurements in the CAP. To apply the model on the field represented in Figure 1, we increased the number of vertices to 1 per $1.4 \mathrm{~m}\left(n_{\text {Egnos }}=1,258\right)$, which represents a measurement rate of $1 \mathrm{~Hz}$ by an operator walking around the field (common practice for verification CAP).

RTK-GPS is a real time surveying method that employs correction signals from a (virtual) base station to solve the integer ambiguities, i.e. the number of integer cycles of $19 \mathrm{~cm}$ that fit along the path between the GPS receiver and the satellite to achieve cm-level accuracy. Several providers, among which 06-GPS in the Netherlands, provide correction signals obtained from a network of fixed base stations (Henry and Polman, 2003).

The company 06-GPS provided us with a time series of 17,570 RTK-GPS positions acquired at $1 \mathrm{~Hz}$ sample rate from their control station in Sliedrecht (almost 5 hours of data). Temporal dependence of the $x$ and $y$ positions were assessed by semivariogram analysis using Gstat. Because of the nature of the data, we assumed no bias in the $x$ and $y$ coordinates (zero mean errors). 
The thus parameterised error model was used to illustrate the positional uncertainty in RTK-GPS measurements. We used the original $(n=14)$ vertices and assumed that the operator walks between the individual measurements at corner points. Each measurement was assumed to take 1 minute.

Topographic map: The BRP (Basis Registatie Percelen) is a Dutch registry of agricultural fields and nature areas. It is largely derived from the Top10Vector digital topographic dataset (Hoogerwerf et al., 2003). Based on Van Buren et al. (2003) we assumed zero mean positional errors in $x$ and $y$ direction $\left(\mu_{X}=\mu_{Y}=0\right)$ for the original $(n=14)$ vertices, with a standard deviation of $2 \mathrm{~m}$ in each direction $\left(\sigma_{X}=\sigma_{Y}=2 \mathrm{~m}\right)$ and no cross correlation. We further assumed that the vertices were digitized by hand at a speed of one per second and that the temporal dependence has a spherical structure with a range of $12 \mathrm{~s}$.

\subsection{Simulation of measured field boundaries}

The Data Uncertainty Engine version 3.0 (DUE) (Brown and Heuvelink, 2007) was used for generating 250 realisations of each of the above described error models (parameterised mpdfs and temporal (cross) correlations). The agricultural field was classified as a deformable object, i.e. the relative positions of the vertices along its boundary can vary under uncertainty.

The coordinates of the vertices were read from a simple time series data file, i.e. one header line specifying the variable names, another line giving the no data values and next 14 (RTK-GPS and topographic map) or 1258 (EGNOS) records each listing date/time, $x$ and $y$ data. DUE 3.0 includes an option to read and write ESRI Shape files, but this functionality currently does not support time series analysis.

In DUE, sampling from the joint-normal distribution is first attempted by factorising the covariance matrix $\Sigma$, giving $L$, such that $\sum=L L^{\mathrm{T}}$, where $\mathrm{T}$ represents the transpose. Secondly, a vector of samples is obtained from the standard Normal distribution $N(0, I)$, with covariance matrix equal to the identity matrix $I$. Sampling from the pdf then involves rescaling by $\mathrm{L}$, and adding the vector of means $\mu$ (Eq. 3):

$$
x=\mu+L z
$$

Where $z$ is a random sample from $N(0, I)$

$x$ is a random sample from the required distribution $N(\mu, \Sigma)$.

If $\Sigma$ is too large to store in memory, or to factorise directly, a sequential simulation algorithm is called from Gstat within DUE (Brown and Heuvelink, 2007).

The parameterised error models were entered as expert judgement on the model page of DUE 3.0. The standard deviation or spread of normally distributed errors $(\sigma)$ was defined by the square root of the sill of the semivariograms. The normal distributions of the coordinates were either centred on the reference coordinates (in case $\mu_{X}=\mu_{Y}=0$ ) or an offset was added to model bias (otherwise). The semivariograms modelled in Gstat were transformed into correlograms, because DUE employs these as the single option of the dependence model. This allows $\sigma$ to vary for each location while the correlogram $(\rho)$ remains a simple function of the absolute (temporal) distance (Brown and Heuvelink, 2007). In case of cross correlations between the $x$ and $y$ errors, the linear model of co-regionalization was used to ensure a valid bivariate covariance structure (Goovaerts, 1997).

\subsection{Effects on field operations}

In this work, we did not consider individual field operations but assumed that a farmer would optimise all field operations (e.g. ploughing, seeding, spraying, harvesting, etc.) based on the mapped field geometry. In this case two types of error may occur: (1) the farmer plans field operations outside the true field; and (2) the farmer sub-utilizes his field because he leaves parts uncultivated. The first type of error may severely harm the environment because agrochemicals may be sprayed into ditches, for example. Both types of error reduce income. We assessed the two types of error by their area by overlaying the realized geometry according to the three error models with the reference geometry depicted in Figure 1. Other losses that may result from sub-optimal planning within the field were not considered.

The 750 realizations of the uncertain time series data produced by DUE were converted to ESRI generate files to create ArcInfo coverages. The topology of the polygons was postprocessed to eliminate any sliver polygon caused by selfintersection of the field boundaries. Next, the realized polygons were intersected with the reference polygon and the statistics of the two types of error were obtained by querying the area attribute from the associated tables. All geo-processing was done in ArcGis 9.1 and Python scripts to allow for looping over the realizations.

\section{RESULTS AND DISCUSSION}

\subsection{Error model}

EGNOS: The EGNOS sample data had biases of $\mu_{X}=0.508 \mathrm{~m}$ and $\mu_{Y}=0.230 \mathrm{~m}$. Figure 2 shows the semivariograms of the EGNOS residuals in $x$ and $y$ direction. The plots were cut at 400 $\mathrm{s}$ because of relatively few data pairs at larger temporal distances. For temporal distances exceeding $300 \mathrm{~s}$ the model fits are poor, but this was assumed to be of little consequence in the subsequent simulations because adjacent vertices are at $1 \mathrm{~s}$ distance. Note also that towards the right the semivariograms are based on fewer data pairs. Based on the semivariograms, the spread of the $x$ and the $y$ residuals were set at $\sigma_{x}=1.16 \mathrm{~m}$ and $\sigma_{y}=1.63 \mathrm{~m}$. We found no evidence for cross correlation. The correlogram $(\rho)$ for both the $x$ and the $y$ residuals was modelled as follows (Eq. 4):

$$
1-\rho=0.132 \operatorname{Sph}(85)+0.792 \operatorname{Gau}(720)+0.076 \operatorname{Per}(220)
$$

where $\operatorname{Sph}(85)=$ spherical structure with range $85 \mathrm{~s}$

Gau $=$ Gaussian structure

$\operatorname{Per}(220)=$ periodic structure with period $220 \mathrm{~s}$

The Gaussian structure with long range was added to improve the fit at larger temporal distances and to bring the standard deviations close to documented values. The cause of the periodic structure is not clear. Periodicity has been observed over short time spans with a static receiver owing to multipath 
effects (Van Willigen, 1995; Amiri-Simkooei and Tiberius, 2007), but in our case time series were based on a moving receiver. Multipath effects happen when a GPS unit receives both the direct GPS signal and signals reflected by e.g. buildings. It differs from site to site and from time to time as it depends on the azimuth and elevation of the satellites and local geometry (Amiri-Simkooei and Tiberius, 2007).

The semivariograms are also notably different from the structure reported by Bogaert et al. (2005). The latter only comprised a single Gaussian structure with an effective range of $30 \mathrm{~s}$ and a rather high sill in comparison to the accuracy that is claimed to be possible with EGNOS augmentation. Obviously, there is no single semivariogram that can be used for all EGNOS enabled GPS receivers under all circumstances. Therefore, our parameterizations of the error model should not be used uncritically beyond the scenarios here presented.
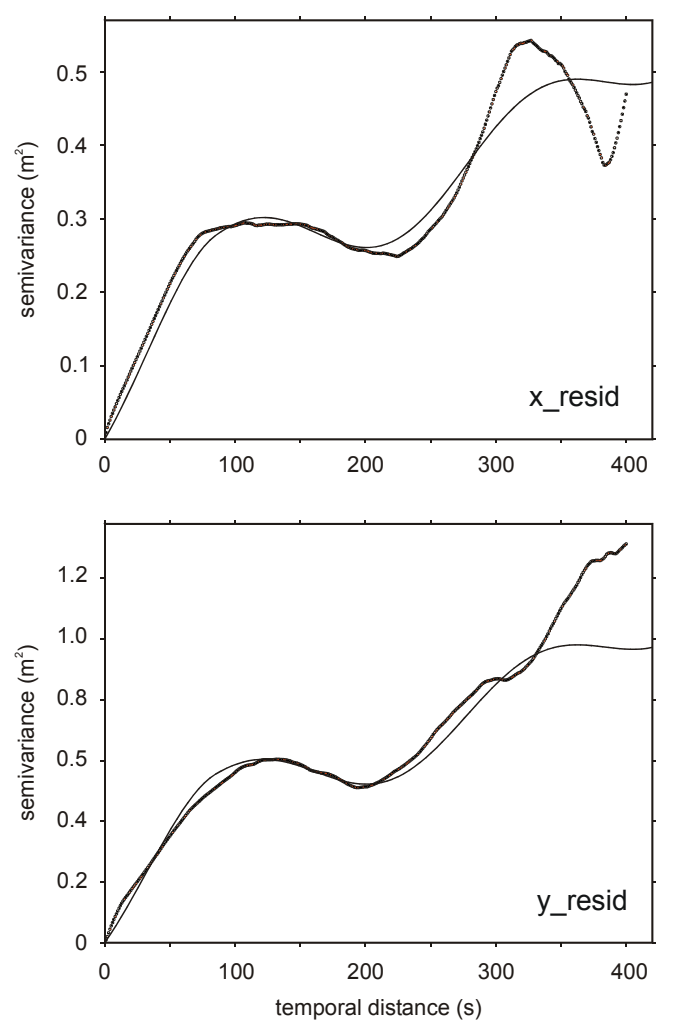

Figure 2. Semivariograms of the EGNOS residuals in $x$ (upper plot) and $y$ (lower plot) direction. Fitted models are indicated by thin lines; the symbols represent experimental data.

RTK-GPS: Figure 3 shows the semivariograms and cross variogram of the RTK-GPS data in $x$ and $y$ direction. The spreads of the $x$ and $y$ errors were set at $\sigma_{x}=0.0061 \mathrm{~m}$ and $\sigma_{y}=$ $0.011 \mathrm{~m}$, respectively and as indicated above, biases were ignored $\left(\mu_{X}=\mu_{Y}=0\right)$. These figures are consistent with RTKGPS accuracies reported elsewhere.. The correlogram for $x$ and $y$ error was modelled by (Eq. 5):

$$
1-\rho=0.041 N u g(0)+0.581 \operatorname{Sph}(500)+0.378 \operatorname{Per}(1250)
$$

Under the linear model of coregionalization, cross correlation was modelled by (Eq. 6):

$$
1-\rho_{x y}=0.0385 \operatorname{Nug}(0)+0.552 \operatorname{Sph}(500)+0.36 \operatorname{Per}(1250)
$$

There is an even more pronounced periodic component in the spatial dependence structures. While such periodicity over short time spans may be attributed to multipath effects (AmiriSimkooei and Tiberius, 2007), its prolonged presence in these data is remarkable. We sub-sampled our time series data to check whether the pattern was caused by some feature of the Gstat software, but the same structure was observed. Possibly, the periodicity is produced by multipath effects somewhere in the RTK network. We will do additional measurements to find out whether the observed pattern is structural.
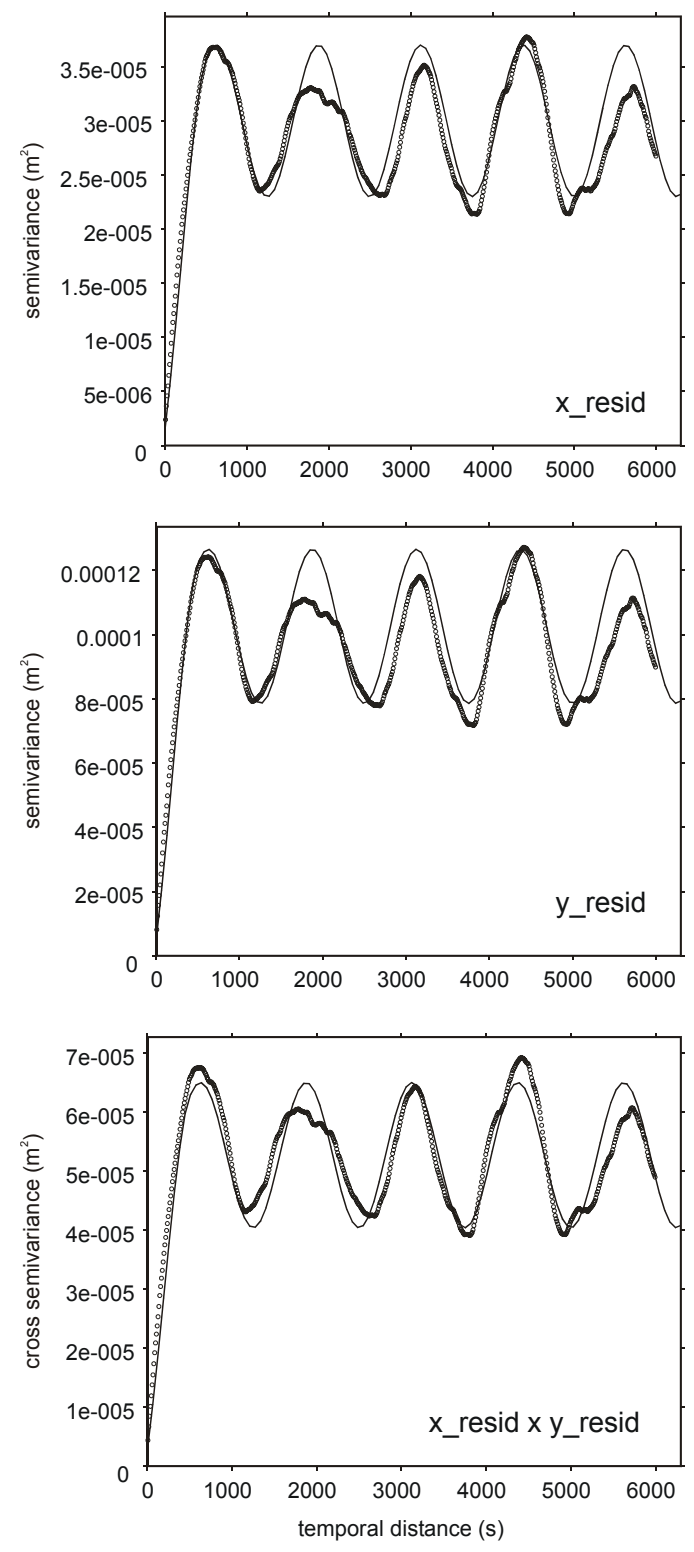

Figure 3. Semivariograms of the $x$ and $y$ RTK-GPS coordinates (upper and middle plots) and cross variogram (lower plot). 


\subsection{Simulation with DUE}

All simulations with DUE 3.0 were performed using the full jpdf, by factorisation of the covariance matrix. In case of the EGNOS scenario (with 1258 vertices), this involved factorisation of a $1258 \times 1258$ matrix (cross-correlations were not accounted for).

\subsection{Effects on field operations}

Figure 4 shows an example realization obtained under the topographic map scenario. If the farmer would rely on this topographic map to plan field operations, in the North he would leave a large strip uncultivated while in the South his plans would cover a ditch. Realizations obtained under the EGNOS scenario (detail shown in Figure 5) give comparable results, but with more irregular field boundaries because of the increased number of vertices. Conversely, on the maps resulting from the RTK-GPS scenario the errors cannot be discerned by the eye unless displayed at a very large scale.

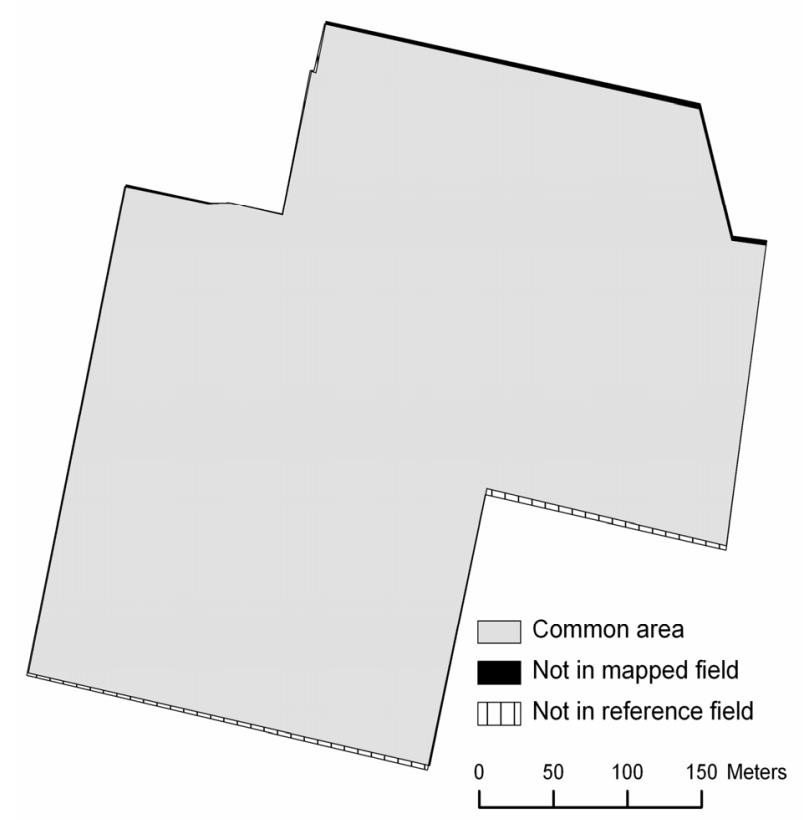

Figure 4. Overlay of reference geometry and an example realization obtained under the topographic map scenario.

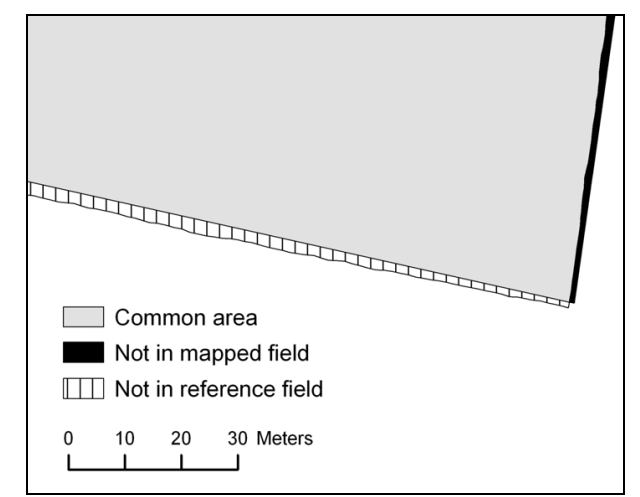

Figure 5. Detail of an overlay of reference geometry and an example realization obtained under the EGNOS scenario, i.e. an operator walking around the field while EGNOS augmented GPS positions are recorded at $1 \mathrm{~Hz}$
Table 1 lists several summary statistics of erroneously mapped areas under our three scenarios. The corresponding histograms are shown in Figure 6. Not surprisingly, the expected incorrectly mapped areas are approximately proportional to the standard deviations of the positional errors for each type of error. On the other hand, the non-Gaussian distributions shown in Figure 6 are symptomatic of the non-linear operation performed on the data. This demonstrates the utility of Monte Carlo simulation, which enables incorporation of operations of any complexity in an error propagation study.

Data such as presented in the percentile columns of Table 1 may be used to assess risks. For example, under the EGNOS scenario there is a probability of $90 \%$ that the area with error type 1 exceeds $469 \mathrm{~m}^{2}$. Whether such risks are acceptable depends on the environmental, financial and other consequences of the errors. In practice, there is some uncertainty surrounding these probabilities, including uncertainty originating from sampling effects and modelling of the joint pdf. While confidence intervals could be computed for these estimates, we do not present them here.

Table 1. Summary statistics of areas in error $\left(\mathrm{m}^{2}\right)$ under three scenarios.

\begin{tabular}{lccccc}
\hline \multirow{2}{*}{ Source } & \multirow{2}{*}{ Error $^{*}$} & \multirow{2}{*}{ Mean } & \multirow{2}{*}{ SD } & \multicolumn{2}{c}{ Percentile } \\
& & & & & \\
EGNOS & 1 & 980 & 435 & 469 & 0.90 \\
& 2 & 1002 & 432 & 485 & 1566 \\
\multirow{2}{*}{ RTK-GPS } & 1 & 6.02 & 3.54 & 2.08 & 10.9 \\
Topographic & 1 & 5.99 & 3.01 & 3.25 & 9.38 \\
map & 2 & 1348 & 685 & 489 & 2299 \\
\hline
\end{tabular}

${ }^{*} 1$ = area included in mapped field, but outside reference field; $2=$ area outside mapped field, but inside reference field.

\section{CONCLUSIONS}

We have demonstrated a general error propagation method that can be used for experimental verification of claims regarding the positional accuracy required for planning field operations based on digital field maps. In our current example we did not plan any field operations (e.g. ploughing, seeding, spraying, harvesting, etc.) directly, but rather assessed the areas where a farmer would erroneously plan activities (while they are outside his field) and areas that would be left without cultivation (while they could be used). The method can easily be adopted to compute error propagation through more complex applications such as path planning for field operations.

We observed periodic components in the temporal dependence structures of the GPS errors. Such periodicity has been attributed to multipath effects over short time spans, but its presence in our experiments and relevance beyond our scenarios require further study.

A definite answer to the question of whether agricultural fields should be measured with cm-level accuracy depends on the environmental and financial consequences of the above described errors and other costs that may occur within the fields. It also depends on the level and type of automation employed by the farmer. Our scenario analysis, nevertheless, showed that for planning and executing field operations a farmer should not blindly rely on approximate field geometry 
as this would leave ample room for accidents (e.g. $90 \%$ chance that an erroneously cultivated area adjacent to the studied field is larger than $469 \mathrm{~m}^{2}$, under the EGNOS scenario).
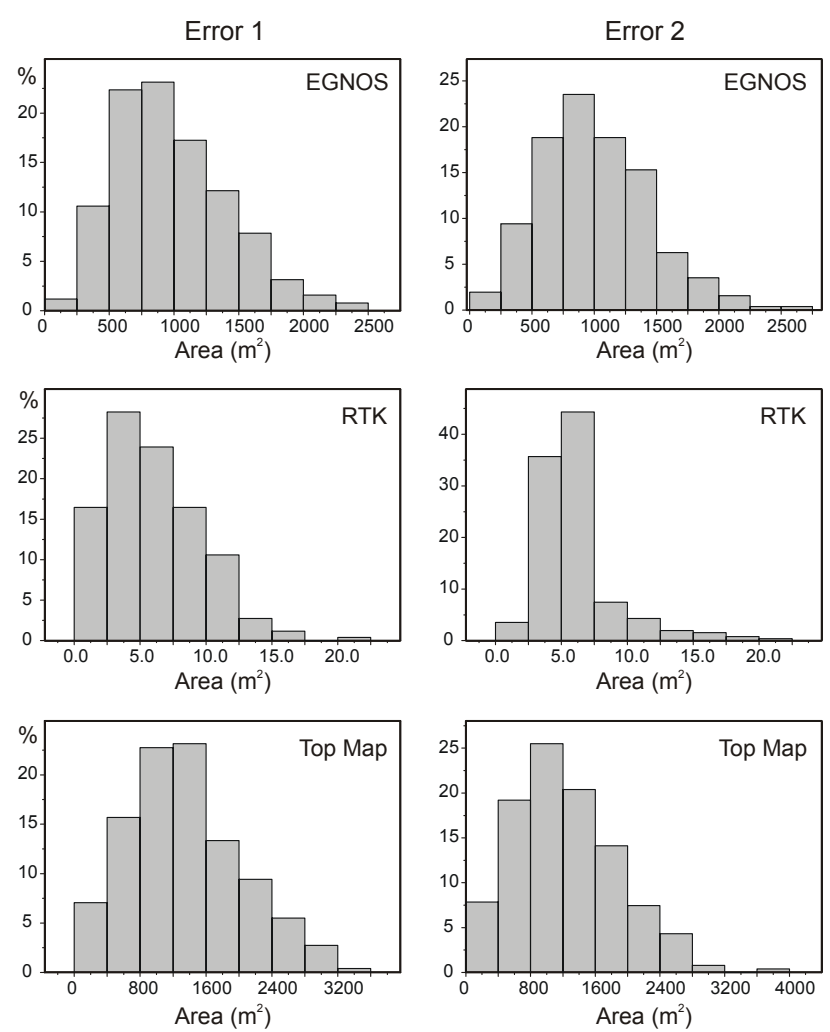

Figure 6. Histograms of areas in error under three scenarios. Types of errors are explained below table 1 .

\section{REFERENCES}

Amiri-Simkooei, A. R. and C. C. J. M. Tiberius, 2007. Assessing receiver noise using GPS short baseline time series. GPS Solutions, 11(1), pp. 21-35.

Atherton, B. C., M. T. Morgan, S. A. Shearer, T. S. Stombaugh and A. D. Ward, 1999. Site-specific farming: A perspective on information needs, benefits and limitations. Journal of Soil and Water Conservation, 54(2), pp. 455-461.

Bogaert, P., J. Delinc and S. Kay, 2005. Assessing the error of polygonal area measurements: a general formulation with applications to agriculture. Measurement Science \& Technology, 16(5), pp. 1170-1178.

Brown, J. D. and G. B. M. Heuvelink, 2007. The Data Uncertainty Engine (DUE): A software tool for assessing and simulating uncertain environmental variables. Computers \& Geosciences, 33(2), pp. 172-190.

Choset, H., 2001. Coverage for robotics - A survey of recent results. Annals of Mathematics and Artificial Intelligence, 31(14), pp. 113-126.

Earl, R., G. Thomas and B. S. Blackmore, 2000. The potential role of GIS in autonomous field operations. Computers and Electronics in Agriculture, 25(1-2), pp. 107-120.
European Space Agency, 2004. "EGNOS." http://esamultimedia.esa.int/docs/br227_EGNOS_2004.pdf (accessed 28-01-2007).

European Space Agency, 2006.. "Egnos system test bed." http://esamultimedia.esa.int/docs/egnos/estb/esaEG/estb.html (accessed 29-01-2007).

Fountas, S., D. Wulfsohn, B. S. Blackmore, H. L. Jacobsen and S. M. Pedersen, 2006. A model of decision-making and information flows for information-intensive agriculture. Agricultural Systems, 87(2), pp. 192-210.

Goovaerts, P., 1997. Geostatistics for Natural Resources Evaluation. New York, Oxford University Press, pp 108-123.

Gunderson, R. W., M. W. Torrie, N. S. Flann, C. M. U. Neale and D. J. Baker, 2000. The collective - GIS and the computercontrolled farm. Geospatial Solutions, July 200, pp. 2-6.

Hekkert, G., 2006. Volautomaten sturen goed - Een prima stuurautomaat is er al voor $€ 7500$. Boerderij. 91, pp. 18-23.

Henry, P. J. A. and J. Polman, 2003. GPS-netwerk operationeel in heel Nederland. Geodesia, 3, pp. 108-114.

Heuvelink, G.B.M., J.D. Brown and E.E. Van Loon, 2007. A probabilistic framework for representing and simulating uncertain environmental variables. International Journal of Geographical Information Science(accepted).

Hoogerwerf, M. R., J. D. Bulens, J. Stoker and W. Hamminga, 2003. Verificatie Kwaliteit BRP gegevensbank. Wageningen, Alterra, Research Instituut voor de Groene Ruimte.

Joint Research Centre, 2005. "2005 GPS Workshop - 5th and 6th October $2005 \quad$ (Wageningen)." http://agrifish.jrc.it/marspac/LPIS/meetings/2005-10-5NL.htm (accessed 29-01-2007).

Keicher, R. and H. Seufert, 2000. Automatic guidance for agricultural vehicles in Europe. Computers and Electronics in Agriculture, 25(1-2), pp. 169-194.

Magellan, 2007. "MobileMapper Pro Specifications." http://pro.magellangps.com/en/products/product_specs.asp?PR ODID=1043 (accessed 29-01-2007).

Nijland, D., 2006. Futuristisch boeren wordt haalbaar, want betaalbaar. VI Matrix, 108, pp. 6-7.

Olynik, M., M. Petovello, M. Cannon and G. Lachapelle, 2002. Temporal impact of selected GPS errors on point positioning. GPS Solutions, 6(1), pp. 47-57.

Pebesma, E. J. and C. G. Wesseling, 1998. Gstat: A program for geostatistical modelling, prediction and simulation. Computers \& Geosciences, 24(1), pp. 17-31.

Tiberius, C., 2003. Handheld GPS receiver accuracy. GPS World, 14(2), pp. 46-51.

Van Buren, J., A. Westerik and E. J. H. Olink, 2003. Kwaliteit TOP10vector - De geometrische kwaliteit van het bestand 
TOP10vector van de Topografische Dienst, Kadaster Concernstaf Vastgoedinformatie en Geodesie.

Van Willigen, D., 1995. Integriteit van (D)GPS signalen. In: Precisie plaatsbepaling met DGPS in Nederland - Kwaliteit, netwerken en toepassingen, Rotterdam, Nederlands Instituut voor Navigatie.

\section{ACKNOWLEDGEMENTS}

This work was partly carried out within the project "Geoinformation requirement for agri-environmental policy" which is co-financed from the program "Space for Geo-information" (Project RGI-017).

We gratefully acknowledge Thales/Magellan and 06-GPS for providing GPS time series, Kemira GrowHow for the aerial photograph used in Fig. 1 and Van Waterschoot Landmeetkunde for the reference geometry. Special thanks go to Aad Klompe (farmer in the Hoeksche Waard and chairman of $\mathrm{H}-\mathrm{WodKa}$ ) who introduced us to the problem setting. 